\title{
Extraction and Characterization of Chitosan from Crustacean Waste: A Constructive Waste Management Approach
}

\author{
P. Premasudha ${ }^{1}$, P. Vanathi ${ }^{2}$, M. Abirami ${ }^{3}$ \\ ${ }^{1}$ Assistant Professor, Department of Nanotechnology, Bharathiar University, Coimbatore - 641 046, India \\ ${ }^{2}$ Assistant Professor, Sengunthar College of Arts and Science, Thiruchengode - 637 205, India
}

${ }^{3} \mathrm{Ph} . \mathrm{D}$. Scholar, PG and Research Department of Microbiology, PSG College of Arts \& Science, Coimbatore - 641 014, India

\begin{abstract}
Biopolymer based products grabbed much attention of researchers today for its various eco and bio friendly properties. From several studies it is understood that ocean ecosystem is one of the major source of biopolymer particularly chitosan. Chitosan is an amino polysaccharide known for its distinctive properties besides biodegradability and biocompatibilities are more imperative. Simultaneously, sea food waste is one of the major issues today and it causes several environmental hazards if not addressed properly. Hence, the crustacean waste is the prime focus of the present study to extract chitosan for various biomedical applications and the extraction was done from shrimp and crab shell waste by chemical method. The process was preceded with several steps like deprotenation, demineralization, decolourization and deacetylation. The extracted chitosan was subjected to several characterizations like physio chemical parameters includes, deacetylation degree, ash content, $\mathrm{pH}$ value, loss on drying and solubility testing. The functional and structural properties were also characterized using XRD and FT-IR respectively. All the characterization data were compared with standard chitosan and confirms the structure of chitosan. Hence, the current study proposed the efficient method of extracting high quality chitosan from shrimp and crab shell waste. This in turn encompasses in proficient crustacean waste management system and keeps the sea food industry associated environment clean.
\end{abstract}

Keywords: Chitin, Chitosan, Extraction, XRD, FT-IR

\section{Introduction}

Globally, sea food attracts the taste buds of many people and its delicacy occupies a prodigious market in an extensive range of products. There is huge number of industrial plants which process the harvested fish. The major focus of fish processing plants is to recover the meat alone not the shells, thereby excretes tons and tons of crustacean by-products as waste. Every year, the shellfish processing plants generates enormous quantity of waste this way and contribute a notable environmental hazard. However the biological wastes are decomposable, the dumping off very huge masses makes the place unhygienic with foul odour and also slow down the process of degradation. Utilization of these dumped crustacean shell waste in a beneficial way by producing value added products is the quick and effective solution to address this problem.

Chitosan, a helical polysaccharide macromolecule found in the exoskeleton of crustaceans such as crabs, shrimp, crawfish, insects, and other arthropods and is the second most abundant natural biopolymer after cellulose. Earlier reports revealed that, both chitin and chitosan have shown to have remarkable biological properties such as bioresorbable degradation products, hydrophilicity, biocompatibility, cellular binding capability and acceleration of wound healing, which accounts for their wide variety of applications in food, cosmetic, biomedical, and pharmaceutical industries [1]. Also the well known physicochemical and impassive functional properties upon modifications, chitosan is well popular in the field of biomedicine and considered as a potential candidate for a wide range of medical applications.
There are various methods available for extraction of chitosan from crustacean wastes but the major disadvantages are time consuming and low yielding. Hence, the current study focuses on simple and efficient method of chitosan extraction.

\section{Materials and Methods}

\section{Sample preparation}

The exoskeleton of shrimp and crab was collected and placed in sealed bags from local fish market of Ukkadam, Coimbatore and refrigerated overnight. Approximately 1500 grams of shrimp exoskeleton was washed and crushed into smaller pieces using a meat tenderizer then allowed to air dry for $3 \mathrm{hrs}$. Air dried samples were transferred to hot air over oven set at $65^{\circ} \mathrm{C}$ for 4 consecutive days to obtain dehydrated material. The dry weights of the samples were determined before going to extract the chitin and prepare chitosan.

\section{Extraction of Chitin and Preparation of chitosan}

1) Deproteinization: Initially, dried shrimp and crab shell waste was treated with $4 \%(\mathrm{w} / \mathrm{v}) \mathrm{NaOH}$ solution at $45^{\circ} \mathrm{C}$ for $24 \mathrm{hrs}$ to remove the protein, separation of alkaliinsoluble fraction was achieved by centrifugation at 4000 rpm for 15 minutes and repeated washing with distilled water was done till the $\mathrm{pH}$ dropped to neutral.

2) Demineralization: Deproteinized shells were treated with $4 \%(\mathrm{v} / \mathrm{v}) \mathrm{HCl}$ solution at $30^{\circ} \mathrm{C}$ for 12 hours to remove minerals, separation of acid-insoluble fraction by 


\section{International Journal of Science and Research (IJSR) \\ ISSN (Online): 2319-7064}

Index Copernicus Value (2015): 78.96 | Impact Factor (2015): 6.391

centrifugation at $4000 \mathrm{rpm}$ for 15 minutes. Further, the separated fraction was washed with distilled water until it is absolutely free of acid, then it was kept for drying at $40^{\circ} \mathrm{C}$ overnight to yield chitin. The obtained chitin hold a slight pink in coloration, hence, before being proceed to chitosan preparation, decolourisation process was done.

3) Decolourisation: Decolourisation was achieved by soaking the obtained chitin in $1 \%$ potassium permanganate for 30 mins followed by $1 \%$ oxalic acid for 30 mins to $2 \mathrm{hrs}$. Finally, the product obtained was designated as purified shrimp and crab shell chitin.

4) Deacetylation: The decolorized chitin was subjected to deacetylation process by treating with $65 \%(w / v) \mathrm{NaOH}$ for 3 days at $30^{\circ} \mathrm{Cto}$ convert as chitosan. The alkali fraction found in chitosan was separated by centrifugation at $4000 \mathrm{rpm}$ for 15 minutes and excess alkali drained off and further washed with distilled water till $\mathrm{pH}$ reaches to neutral. Obtained chitosan fraction was dried at $40^{\circ} \mathrm{C}$ for overnight and stored at room temperature until further exploration.

\section{Properties of Chitosan}

\section{Degree of deacetylation}

Degree of deacetylation refers to the removal of acetyl group from the chain which is determined by potentiometric titration. Homogenous solution of chitosan was prepared using diluted $\mathrm{HCl}(0.010 \mathrm{~mol} / \mathrm{L})$ which was titrated against $0.1 \mathrm{M} \mathrm{NaOH}(\mathrm{w} / \mathrm{v})$. The end point is determined by the inflections of the $\mathrm{pH}$ values. Two inflections were mainly considered out of which first one corresponds to neutralization of $\mathrm{HCl}$ and second one neutralization of ammonium ions from chitosan. The difference between two points gives the amount of amino groups in the chitosan it was also referred as degree of deacetylation [2].

$$
D D \%=100-\mathrm{DA} \%
$$

DD represents- Degree of Deacetylation

\section{pHvalue}

The $\mathrm{pH}$ measurement of chitosan solutions were carried out using $\mathrm{pH}$ meter with microprocessor.

\section{Ash value}

To determine the ash value of extracted chitosan, $2.0 \mathrm{~g}$ of chitosan sample was placed into previously ignited, cooled, and tarred crucible. The samples were heated in a muffle furnace preheated to $650^{\circ} \mathrm{C}$ for $4 \mathrm{hrs}$. The crucibles were allowed to cool in the furnace to less than $200^{\circ} \mathrm{C}$ and then placed into desiccators with a vented top [3]. Percentage of ash value is calculated using the following formula,

\section{Loss on drying}

$$
\text { Ash }(\%)=\frac{\text { weight of residue }, g}{\text { weight of sample }, g} \times 100
$$

Loss on drying of the prepared chitosan was determined by the gravimetric method. The water mass loss was determined by drying the sample to constant weight and measuring the sample before and after drying [3]. The water mass (or weight) was the difference between the weights of the wet and oven dry samples.

$$
\text { Loss on drying }(\%)=\frac{\text { wet weight-dry weight }}{d r y \text { weight }} X 100
$$

\section{Solubility of Chitosan}

To estimate the solubility nature of extracted chitosan was determined according to Fernandez-Kim [4]. About 0.1g of chitosan powder sample was taken in centrifuge tube and dissolved in $10 \mathrm{ml}$ of $1 \%$ acetic acid and kept in incubated shaker $(250 \mathrm{rpm})$ at $25^{\circ} \mathrm{C}$ for 30 minutes. The solution was immersed in boiling water bath for 15 minutes and cooled to room temperature followed by centrifuge at $12,000 \mathrm{rpm}$ for 7 minutes and the supernatant was discarded. The undissolved particles were thoroughly washed using distilled water by centrifuging the contents at $10,000 \mathrm{rpm}$ for 10 minutes and the supernatant was discarded. The undissolved pellets were dried at $70^{\circ} \mathrm{C}$ for 24 hours. At the end the dried particles were weighed and the solubility percentage was calculated as,

Solubility $(\%)=($ initial weight of tube + chitosan $)-($ final weight of tube + chitosan) $\times 100$

\section{Characterization of Chitosan}

\section{FT-IR analysis}

The chitosan samples were characterized from 4000 to 400 $\mathrm{cm}^{-1}$ with $\mathrm{KBr}$ pellets using infrared spectrophotometer to identify the functional groups of extracted chitosan and the groups were confirmed by FTIR analysis. In this study, the IR spectra of the extracted chitosan samples of crustaceans were analyzed and compared with the IR spectrum of commercial chitosan.

\section{XRD measurements}

The extracted chitosan samples were subjected to XRD measurements with $2 \theta$ angle from $20^{\circ}$ to $80^{\circ}$ at $0.02 \mathrm{deg}$ min149 ${ }^{-1}$, with $2 \theta$ time constant. Briefly, PAN ANALYTICAL X-ray diffractometer machine operating at a voltage of $40 \mathrm{kV}$ and current of $20 \mathrm{~mA}$ with $\mathrm{Cu} \mathrm{K}(\alpha)$ radiation of $1.54187 \mathrm{~nm}$ wavelength was set and the results were measured.

\section{Results and Discussion}

\section{Extraction of chitin and preparation of chitosan}

\section{Extraction of chitosan from crustaceans}

In present study, the total yield of chitosan obtained from crustaceans (shrimp and crab) is showed in Figure 1. Results of the present study clearly evidenced that, the maximum yield of chitosan was obtained from shrimp shell waste was recorded as $46 \%$. These results are in agreement with the earlier reports of Puvvada et al. [3] who reported that, the yield of chitosan from shrimp shells was about $34 \%$. Moreover, in the present study obtained yields of chitosan is higher than the reported methods, this could be explained by the nature of environment and divergence in shrimp species and also method of extraction.

Other hand, $34 \%$ of total chitosan yield was recorded with crab shell waste. These results are in line with the earlier report of Yen et al. [5] who reported the yield of chitosan obtained from crab shell is varied in a range of (30-36.7\%), and also demonstrated that, the variation in the yield can be explained by the difference in the species being used and reaction time which also has a positive effect on the yield. In 


\section{International Journal of Science and Research (IJSR) \\ ISSN (Online): 2319-7064}

Index Copernicus Value (2015): 78.96 | Impact Factor (2015): 6.391

the current study, the higher yield of chitosan was due to the repetition of deprotonation and demineralization process resulted in maximum yield. The yield also depends on the concentration of acid and alkali and its reaction time with the chitin substrate.

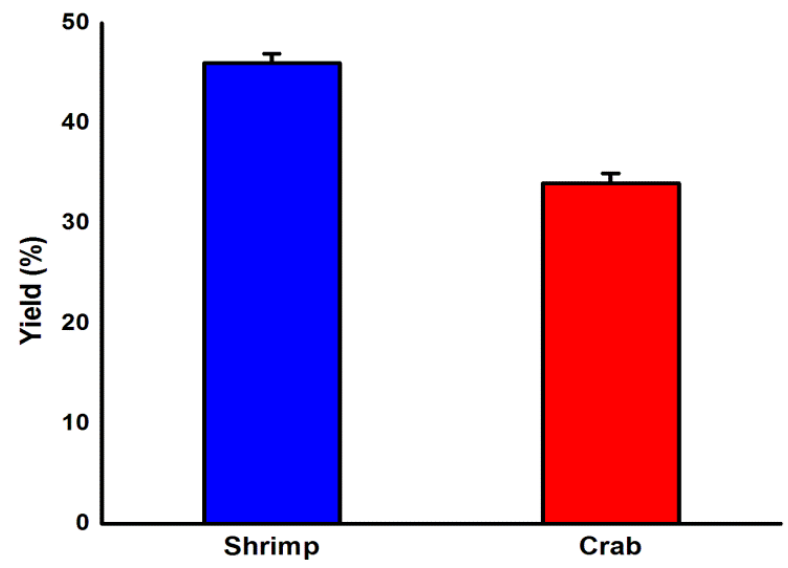

Figure 1: The yield percentage of chitosan extracted from crustaceans

Table 1: The properties of chitosan extracted from crustaceans and fungal biomass

\begin{tabular}{|c|c|c|c|}
\hline Characteristics & Standard chitosan & Shrimp & Crab \\
\hline Degree of deacetylation & 99.99 & 88.48 & 87.20 \\
\hline pH value & 7.2 & 6.8 & 7.8 \\
\hline Ash value (\%) & 1.16 & 1.86 & 1.45 \\
\hline Loss on drying (\%) & 9.75 & 8.0 & 9.24 \\
\hline $\begin{array}{c}\text { Solubility (\%) in Acetic } \\
\text { acid }\end{array}$ & 99 & 98.25 & 98.75 \\
\hline
\end{tabular}

\section{Properties of chitosan}

\section{Degree of deacetylation (DD)}

The degree of deacetylation (DD) of the total chitosan obtained from both crustaceans shown in Table 1. The shrimp chitosan showed maximum DD value of $88.48 \%$ whereas the minimum DD observed for the chitosan extracted from crab was observed as $80.12 \%$.The DD denotes the removal of acetyl group from the long chain of chitin and it plays a substantial role in deciding the precise application of chitosan. The DD is an important parameter to be considered for physical and chemical properties of chitosan including solubility, adsorption, chemical reactivity, covalent linking, encapsulation and biodegradability [3]. DD of chitosan may range from $30 \%$ to $95 \%$ depending on the available source and procedure [6]. According to Pochanavanich and Suntornsuk [7], the degree of deacetylation depends on the source of chitin, concentration of acid and alkaline used, time and temperature etc. However, Puvvada et al. [3] depicted that the DD values with higher range are the consequence of the high amount of protein, which in turn yields chitosan with superior quality suitable for various pharmaceutical applications.

\section{Determination of $\mathbf{p H}$ value}

In the present study (Table 1) shows, the determined $\mathrm{pH}$ values of the prepared chitosan were varied from the $6.8-$ 7.8 and obtained results were in line with the earlier reports of Sneha et al. [8] who reported the $\mathrm{pH}$ variation of chitosan obtained from crustaceans from species to species. The $\mathrm{pH}$ value of chitosan plays a major role in functional properties of chitosan including antimicrobial, cyototoxicity and also indirectly influences the hydrophilicity and deacetylation ratio.

\section{Determination of Ash value}

The results of ash content present in chitosan extracted from crustaceans and fungi are listed in Table 1 and the results of the present study were revealed that, almost all the samples are close to the range of standard values. The ash content of chitosan is an indication of the effectiveness of the method employed for removing inorganic materials. The determined maximum ash content of $2 \%$ in shrimp is due to the presence of calcium carbonate which is found in large amount in shrimp shells and is lowest in squid pen chitosan, about $0.17 \%$ [9]. These results in agreement with the previous reports of Sneha et al. [8] who reported the low ash value of $1.86 \%$ of shrimp chitosan. On the other hand, chitosan extracted from crab produced $1.45 \%$ in the present study. Interestingly, Cho et al. [10] reported commercial chitosan to have ash value about $1.18 \%$ and these values are in line with the obtained results of the present study.

\section{Determination of dry weight}

Present study reveals that, loss of moisture content in studied chitosan of shrimp and crab ranging from $8.0 \%-9.24 \%$ of total weight respectively. These results are in well agreement with the reports of Sneha et al. [8], who explained the acceptable moisture content of chitosan powder should be $<10 \%$ for commercial applications. The reports of Tajik et al. [11] also states that chitosan from shrimp shell contains moisture in the range of $1.0-1.30 \%$ which in turn depends upon the season, relative humidity and intensity of sunlight of the raw material source.

\section{Solubility determination}

The results of chitosan solubility are showed in Table 1 which clearly reveals the high solubility nature of chitosan in $1 \%$ acetic acid aqueous solution. The solubility of chitosan is one of the important parameters for quality of chitosan, where higher solubility will produce a better chitosan. There are several critical factors affecting chitosan solubility including temperature and time of deacetylation, alkali concentration and prior treatments applied to chitin isolation, ratio of chitin to alkali solution and particle size. The solubility, however, is controlled by the degree of deacetylation and it is estimated that deacetylation must be at least $85 \%$ complete in order to achieve the desired solubility [12]. Proportionally increase in solubility was observed with increasing deacetylation degree. Brine and Austin, [13] suggested that the incomplete removal of protein and acetyl group leads to lower solubility. Since solubility of chitosan depends on the removal of acetyl group from chitin, therefore the lower DD value could adversely interfere with the results. Chitosan, unlike chitin has high content of highly protonated free amino group that very well attracts ionic compounds. This could be the reason for its solubility in mild inorganic acid [14]. 


\section{International Journal of Science and Research (IJSR) \\ ISSN (Online): 2319-7064}

Index Copernicus Value (2015): 78.96 | Impact Factor (2015): 6.391

\section{Fourier Transform Infrared Spectroscopy (FTIR)}

FTIR results clearly indicated the presence of functional groups including amino and other hydroxyl groups as like commercial chitosan. The results of the FTIR spectra of chitosan prepared from the different sources were depicted in Figure 2 and table 2. The peaks at $3318 \mathrm{~cm}^{-1}, 3332 \mathrm{~cm}^{-1}$ and $3357 \mathrm{~cm}^{-1}$ of $\mathrm{NH}_{2}$ stretching belongs to the functional groups of primary amines of commercial chitosan, shrimp chitosan and crab chitosan respectively. The bands at 2813 $\mathrm{cm}^{-1}, 2826 \mathrm{~cm}^{-1}$ and $2826 \mathrm{~cm}^{-1}$ of $\mathrm{OH}$ stretching of carboxylic acids were observed in all chitosan samples. The peaks at $1423 \mathrm{~cm}^{-1}, 1409 \mathrm{~cm}^{-1}$ and $1397 \mathrm{~cm}^{-1}$ of commercial chitosan, shrimp chitosan and crab chitosan indicate the C-N stretch of secondary amine group. The functional group of nitro compounds of N-O symmetric stretch of peaks at 1358 of commercial chitosan were shifted to $1345 \mathrm{~cm}^{-1}$ of shrimp and crab chitosan. The bands at $1137 \mathrm{~cm}^{-1}, 1072 \mathrm{~cm}^{-1}$ and $1111 \mathrm{~cm}^{-1}$ of $-\mathrm{NH} 2$ stretch of free amine groups was observed in all the samples. The functional group of aromatic amines of $\mathrm{C}-\mathrm{N}$ stretch was observed in the entire chitosan sample at $1280 \mathrm{~cm}^{-1}$. The primary and secondary amines of N-H stretch were observed at the peaks of 743 $\mathrm{cm}^{-1}, 747 \mathrm{~cm}^{-1}$ and $902 \mathrm{~cm}^{-1}$ in commercial chitosan, shrimp chitosan and crab chitosan respectively. The same FTIR results of chitosan are also observed by Zvezdova [15].

Table 2: FTIR spectra of commercial chitosan, extracted shrimp chitosan and crab chitosan

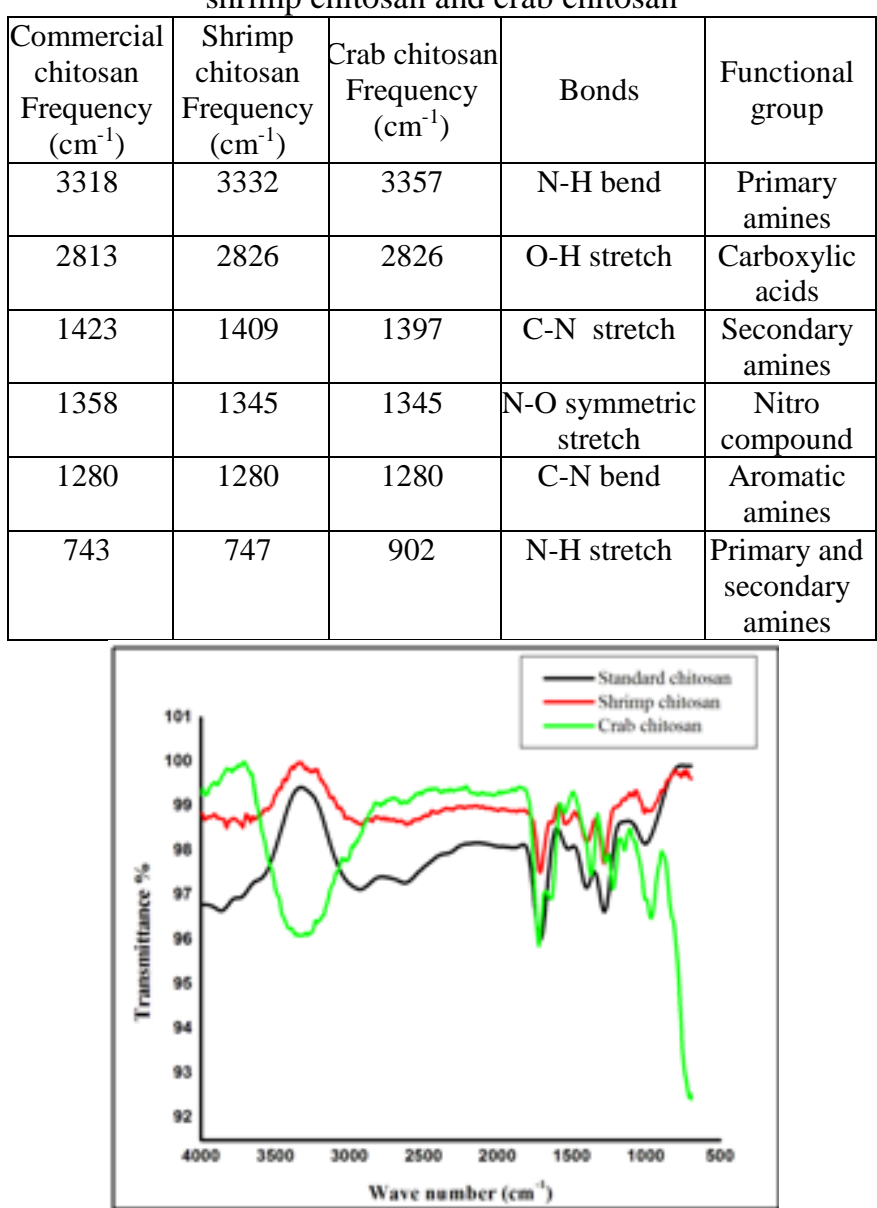

Figure 2: FTIR spectral characterization of chitosan extracted from Shrimp and crab

\section{X-ray diffraction (XRD)}

XRD results clearly indicated the presence of chitosan peaks was compared by de Queiroz et al. [16]. The results of the XRD spectra of chitosan prepared from the different sources were depicted in Figure 3 and 4. The XRD pattern of shrimp chitosan showed characteristic peaks at $2 \theta=9.10^{\circ}, 20.10^{\circ}$ in $2 \theta=9.9-10.30,19.8-20.4$ and crab chitosan shows $2 \theta=$ $9.0^{\circ}, 20.30^{\circ}$ in $2 \theta=9.710-10.50,18.90-20.180$. The sharper peaks are an evidence of semi - crystalline structure.

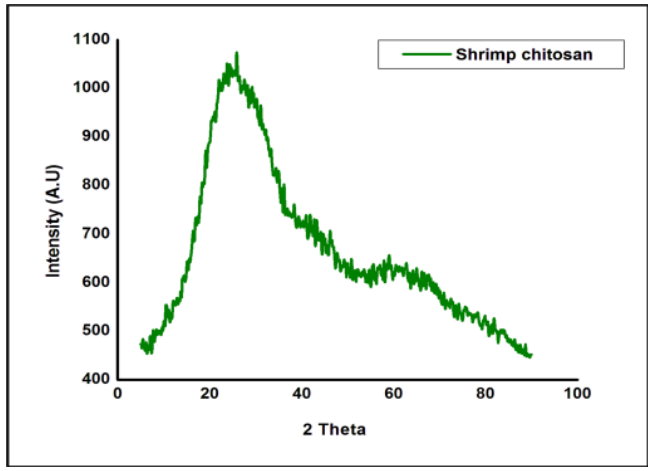

Figure 3: Spectral characterization of chitosan extracted from Shrimp

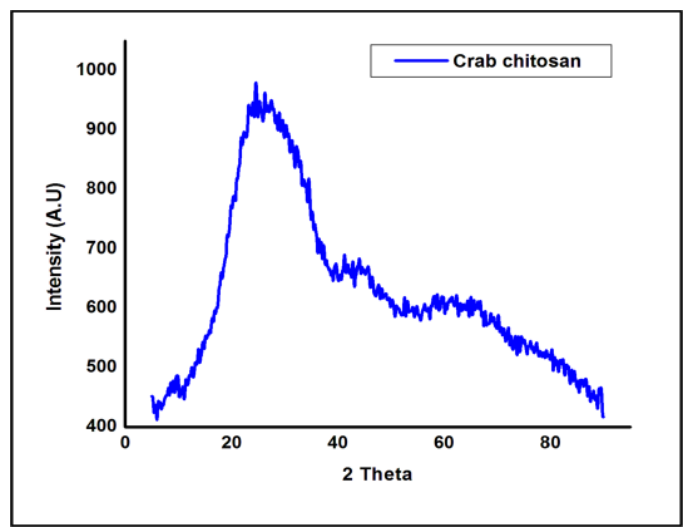

Figure 4: Spectral characterization of chitosan extracted from $\mathrm{Crab}$

\section{Conclusion}

In the current scenario many industries like health care, biomedical, pharmaceutical etc. is in need of high quality, biocompatible and biodegradable materials like chitosan for addressing many health care issues. At the same time there are industries like fish processing plants generates huge quantity of crustacean wastes which causes environmental hazard. Fortunately, these bio wastes are considered as a potent source of chitosan. Keeping all these aspects in my mind the current research work is done to extract natural chitosan from crustacean wastes which are obtained from fish market out fills. Chitosan was extracted by adopting modified process of previous methods and the yield was also high due to the repeated process of deprotonation and demineralization steps. The physio-chemical parameters and structural characteristics are in agreement with commercial chitosan standard. To specific, the obtained chitosan had high deacetylation degree (DD), good solubility and a dense crystalline structure which has greater scope in various industrial applications as well. 


\section{Future Scope of the Work}

The study focuses mainly on the insights of dual beneficiary scheme which includes efficient waste management process and extraction of chitosan from sea food waste. Chitosan is one of the notable compounds for many researchers in the field of biomedical, pharmaceutical sciences and also in various fields of biotechnology. In global scenario, the chitosan and its derivatives have greater scope in biomedical industry and the current study focused on the better utilization of natural resources.

\section{References}

[1] D.W. Lee, H. Lim, H.N.Chong, W.S. Shim. "Advances in chitosan material and its hybrid derivatives: a review," The Open Biomaterials Journal, 1(1), pp. 10 20, 2009.

[2] Y. Zhang, X. Zhang, R. Ding, J. Zhang, J. Liu. "Determination of the degree of deacetylation of chitosan by potentiometric titration preceded by enzymatic pretreatment", Carbohydrate Polymers, 83(2), 813 - 817, 2011.

[3] Y.S. Puvvada, S. Vankayalapati, S. Sukhavasi. "Extraction of chitin from chitosan from exoskeleton of shrimp for application in the pharmaceutical industry," International Current Pharmaceutical Journal, 1(9), pp. 258 - 263, 2012.

[4] S.O. Fernandez-Kim. Physicochemical and functional properties of crawfish chitosan as affected by different processing protocols, 2004.

[5] M.T. Yen, J.H. Yang, J.L. Mau. "Physicochemical characterization of chitin and chitosan from crab shells" Carbohydrate Polymers, 75(1), 15 - 21, 2009.

[6] A. Di Martino, L. Liverani, A. Rainer, G. Salvatore, M. Trombetta, V. Denaro. "Electrospun scaffolds for bone tissue engineering," Musculoskeletal surgery, 95(2), pp. 69 - 80, 2011.

[7] P. Pochanavanich, W. Suntornsuk. "Fungal chitosan production and its characterization," Letters in applied microbiology, 35(1), pp. 17 - 21, 2002.

[8] Sneha Paul, Aishwarya Jayan, Changam Sheela Sasikumar, Sanjay M Cherian. "Extraction and purification of chitosan from chitin isolated from sea prawn (Fenneropenaeus indicus)," Asian Journal of Pharmaceutical and Clinical Research, 7(4), pp. 201 204, 2014.

[9] R. Shepherd, S. Reader, A. Falshaw. "Chitosan functional properties," Glycoconjugate journal, 14(4), pp. 535 - 542, 1997.

[10] Y.I. Cho, H.K. No, S.P. Meyers. "Physicochemical characteristics and functional properties of various commercial chitin and chitosan products," Journal of Agricultural and Food Chemistry, 46(9), pp. 3839-3843, 1998.

[11]H. Tajik, M. Moradi, S.M.R. Rohani, A.M. Erfani, F.S.S. Jalali. "Preparation of chitosan from brine shrimp (Artemia urmiana) cyst shells and effects of different chemical processing sequences on the physicochemical and functional properties of the product," Molecules, 13(6), pp. 1263 - 1274, 2008.

[12]H.K. No, S.P. Meyers. "Preparation and characterization of chitin and chitosan - a review,"
Journal of aquatic food product technology, 4(2), pp. 27 $-52,1995$.

[13] C.J. Brine, P.R. Austin. "Chitin variability with species and method of preparation," Comparative Biochemistry and Physiology Part B: Comparative Biochemistry, 69(2), pp. 283 - 286, 1981.

[14] A.T. Biswas, C. Gargi. "Extraction of Chitosan from Prawn Shell Wastes and Examination of its Viable Commercial Applications," International Journal on Theoretical and Applied Research in Mechanical Engineering, 2 (3), pp. 17 - 24, 2013.

[15]D. Zvezdova. "Synthesis and characterization of chytosan from marine sources in Black Sea. Annual Proceedings," Angel Kanchev" University of Ruse, 49(9.1), pp. 65 - 69, 2010.

[16] R.S.C.M. de Queiroz Antonino, B.R.P. Lia Fook, V.A. de Oliveira Lima, R.Í. de Farias Rached, E.P.N. Lima, R.J. da Silva Lima, M.V. Lia Fook. "Preparation and Characterization of Chitosan Obtained from Shells of Shrimp (Litopenaeus vannamei Boone)," Marine drugs, 15(5), pp. 141, 2017

\section{Author Profile}

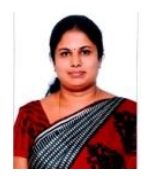

Dr. P. Premasudha currently working as Assistant Professor in the Department of Nanoscience and Technology, Bharathiar University, Coimbatore 46 and her area of specialization is Bio compounds and its applications and Nanobiotechnology. She is having about 13 years of teaching and research experience in the field of Microbiology. She has completed her Doctorate in Microbiology and she has filed a patent under Indian Patent agency in 2015 for the development of a novel wound dressing material. She is also running a research project worth Rs.14.9 lakhs funded by DRDO, New Delhi and the main theme of the proposal is to isolate the pigments from microorganisms as a natural colourant. She has authored a book chapter and also published several articles in various National and International peer reviewed journals. She has guided M.Sc, M.Tech and M.Phil students and she is a Life member in Asian Polymer Association (APA). She has cleared State Level Eligibility Test (SET) in 2006 in Life Science. She had worked in several capacities from Lecturer to Head of the department and gained an experience as active member in academic affairs.

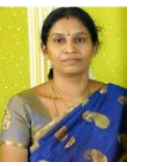

Dr. P. Vanathi, Head and Assistant Professor Department of Biotechnology Sengunthar Arts \& Science college, Tiruchengode. She has an academic expertise of 13 years from the year 1997, completed the doctoral degree in Microbiology. Nearly 9 M.phil scholars have been produced so far. Her area of specialization is Microbial Biotechnology.

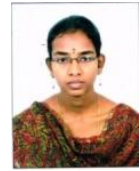

M.Abirami, pursuing Ph.D. in Microbiology in PG and Research Department of Microbiology, PSG College of Arts and Science, Coimbatore. Her area of specialization is medical textile microbiology and Nanotechnology. 